\title{
Etching patterns on the monoclinic and orthorhombic paracetamol
}

\author{
M.A. Mikhailenko, ${ }^{1,3}$ T.N. Drebushchak, ${ }^{1,2,3}$ T.P. Shakhtsneider, ${ }^{1,2}$ \\ and V.V. Boldyrev ${ }^{1,2,3 *}$ \\ ${ }^{1}$ Research and Education Center 008 (MDEST), Novosibirsk State University, Pirogova, \\ 2, Novosibirsk, 630090, Russia \\ ${ }^{2}$ Institute of Solid State Chemistry, Russian Academy of Sciences, Kutateladze, 18, \\ Novosibirsk, 630128, Russia \\ ${ }^{3}$ Novosibirsk State University, Pirogova, 2, Novosibirsk, 630090, Russia \\ E-mail:boldyrev@,nsu.ru
}

\section{Dedicated to Professor Alexander I. Konovalov on the occasion of his $70^{\text {th }}$ anniversary}

(received 01 Oct 04; accepted 03 Feb 05; published on the web 03 Mar 05)

\begin{abstract}
The initial stages of etching the monoclinic (I) and the orthorhombic (II) polymorphs of paracetamol crystals by a series of agents (10\%-solutions of acetic anhydride, pyridine, 1,4dioxane, morpholine, N-methylmorpholine in carbon tetrachloride) were studied and the corresponding etching patterns were compared. For the first time, it was possible to observe different etching patterns when dissolving not only the faces of the crystals parallel to molecular layers, but also the faces normal to the molecular layers.
\end{abstract}

Keywords: Paracetamol, etching pits, hydrogen bonds, molecular recognition

\section{Introduction}

The dissolution of crystals is a complex heterogeneous catalytic process, which attracts much attention because of its importance for many practical applications. ${ }^{1}$ It is also of fundamental interest. The etching patterns observed at the initial stages of the dissolution of crystals are a manifestation of the anisotropy of the crystal structure and of the interactions in this structure. ${ }^{1-4}$ The etching patterns result from a complex interplay of several stages of the dissolution process. The factors affecting the shapes of the etching pits formed at the initial stages of dissolution were studied in details for the halides of alkali metals, silicon, or $\mathrm{A}^{\mathrm{III}} \mathrm{B}^{\mathbf{V}}$ compounds. Still, even for the best studied systems, there is no universally applicable theory that could explain all the 
experimentally observed effects and make reliable predictions on the location of etching pits, on their growth rates, and, especially, on their shapes. ${ }^{1}$

The location of the etching pits is usually correlated with the defect sites in the crystals most commonly, with dislocations, but also with surface defects, vacancy or impurity clusters. "Crystallographic etching pits" were also described. Their location does not always coincide with the dislocations, and their walls are formed by faces with low hkl - indices. The shapes of the crystallographic etching pits were correlated with the anisotropy of crystal structure. It is known that the shapes of the etching pits can be different not only when etching different faces of the same crystal using the same etching agent, but also when etching the same face with the solutions of the same etching agent of different concentrations, or containing different 'poison' impurities. No general explanation of these phenomena was suggested, but it could be noticed that any changes in the shapes of etching patterns were usually observed, when chemical reaction(s) were involved in the etching process. ${ }^{1}$

The dissolution and growth of paracetamol (to be more precise - of its more stable monoclinic polymorph) were described repeatedly in many publications. ${ }^{4}$ This system is important for the pharmaceutical industry, but also for fundamental research. A molecule of paracetamol (Figure 1) has a proton-donor $\mathrm{NH}$-group, a proton-acceptor $\mathrm{C}=\mathrm{O}$-group, whereas the $\mathrm{OH}$-group can act simultaneously as a proton-donor and a proton-acceptor.

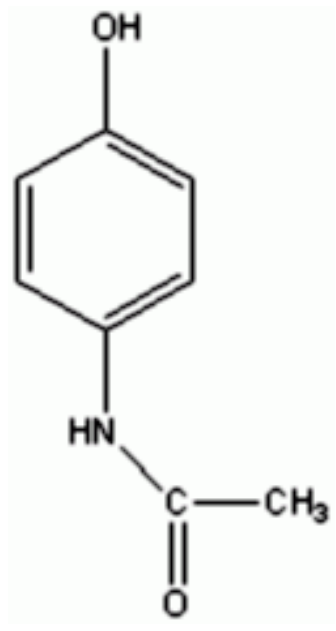

Figure 1. Paracetamol molecule.

In the crystalline state, the molecules of paracetamol are linked by $\mathrm{NH} \ldots \mathrm{O}$ and $\mathrm{OH} \ldots \mathrm{O}$ hydrogen bonds into chains. The chains are further linked into two-dimensional networks, which, in turn, give 3-D layered structures with the van der Waals interactions between the layers. The chains are topologically the same in paracetamol I and paracetamol II, but the way how the chains are linked into the layers is different (Figure 2). ${ }^{5,6,7,8}$ 


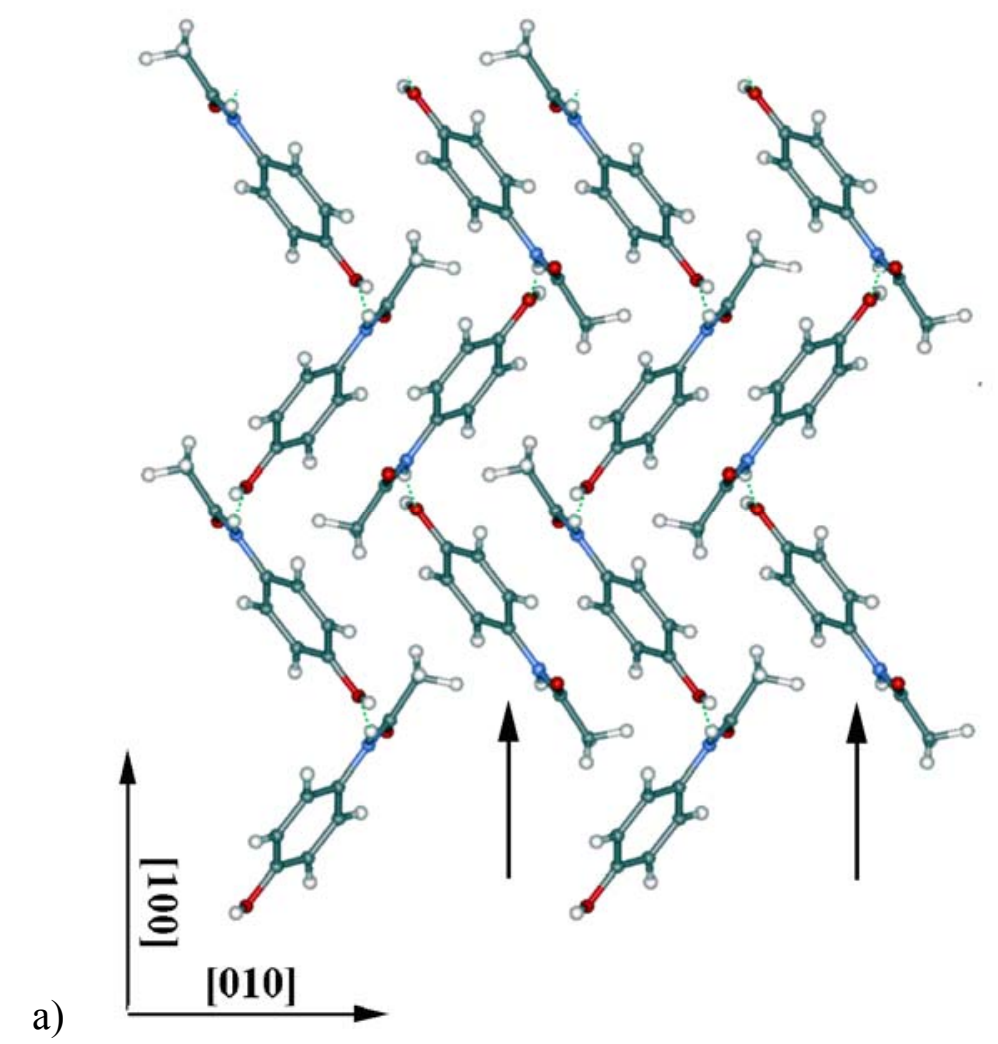

b)

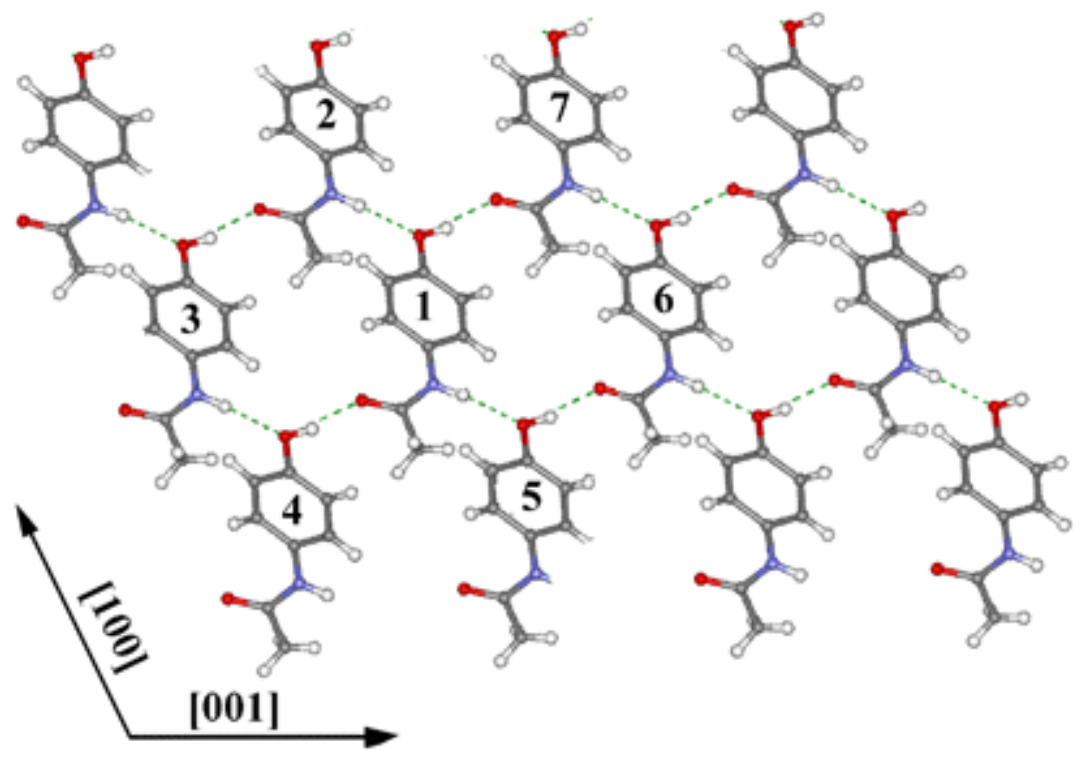

Figure 2. Projection of the paracetamol I structure (space group P21/a): a) (001)-face, b) (010)face. The numbering of molecules is as used for the intermolecular distance calculations in Table 1 (see below). 
a)
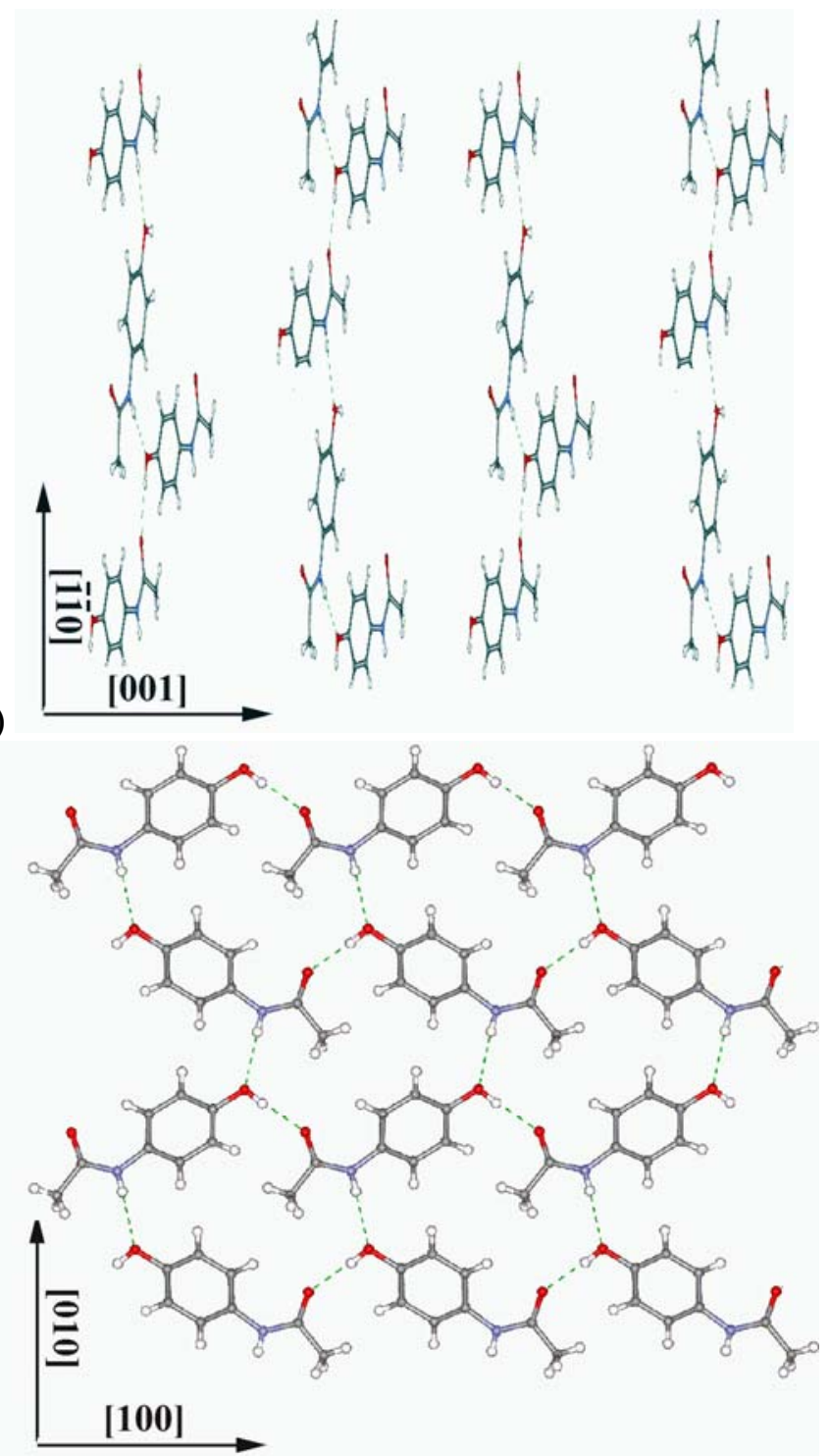

b)

Figure 3. Projection of the paracetamol II structure (space group Pbca): a) (110)-face (molecules are tilted with respect to the projection plane), b) (001)-face.

The faces of paracetamol differ in the surface energy and on their interaction with solvents, due to the different packing densities of molecules, and also because different functional groups are exposed to the solvent molecules at different faces. ${ }^{9}$ This manifests itself in the different growth rates of different faces, or in their different wettability, which were studied for paracetamol I. . $^{2,3,9,10,11,12}$

In a series of our earlier publications we have studied (by optical microscopy ${ }^{2,3,10,11,12}$ and by the $\mathrm{AFM}^{13}$ ) the etching patterns formed on different faces of paracetamol I at the initial stages of the dissolution in different solvents (dichloroethane, nitrobenzene, acetic anhydride, acetone, ethyl acetate, and pyridine in carbon tetrachloride), and also, for a comparison, as a result of thermal treatment (sublimation). ${ }^{2,3}$ The shapes of the etching pits formed on different crystal 
faces were shown to be different for the same etching agent, and also for the chemical ${ }^{10,11,12}$ and for the thermal etching. ${ }^{2,3}$ The etching patterns on the same face (010), parallel to the molecular layers, were shown to be different for different etching agents. ${ }^{2,3,10,11,12}$ A correlation between the donor numbers of etching agents and the extent of the anisotropy of the etching pits (from lines alongated along one crystallographic direction to almost isometric parallelograms and hexagons) was suggested. ${ }^{10}$ The cooperative nature of the dissolution with a positive feed-back accounting for the autolocalization of the process was discussed. ${ }^{11}$ The structure of the hydrogen-bonded 2D-network and the mechanism of the selective interaction of the solvent molecules with different functional groups of the paracetamol molecules at the crystal surface were considered when interpreting the etching patterns. ${ }^{12}$ As additional arguments in favor of the interpretation proposed in ref., ${ }^{3}$ the anisotropy of structure compression on cooling ${ }^{14}$ and with increasing hydrostatic pressure ${ }^{8,15,16,17,18}$ were considered.

Several years later, the authors of ref. ${ }^{19}$ of have completely reproduced all the experimental results published in refs., ${ }^{2,3,10,11,12}$ using exactly the same set of solvents and the same experimental techniques (although, strangely, they did not cite any of the previous publications). They have suggested another interpretation of these results, assuming that it is the surface diffusion that plays the key role in forming etching patterns. To prove the validity of their model, they have carried out a computer simulation of the dissolution process (in a rather rough approximation, the validity of the main postulates of the applied model being not unambiguous). The agreement between the experimental and the simulation results was rather poor: for several etching agents the etching patterns could not be reproduced by the simulation. To explain the discrepancy, the above authors ${ }^{19}$ were forced to take into consideration the same factors as we did in our previous publications, 2,3, 11,12 namely the anisotropy of the crystal structure, the differences in the interaction of solvent molecules with different functional groups at the paracetamol crystal surface (molecular recognition between solvent molecules and crystal molecules), and the changes or the interruption of the original hydrogen-bonded 2D-network in the paracetamol crystals resulting from an adsorption of a solvent molecule.

All the above mentioned factors can be important for the dissolution. To study their relative inputs into the formation of the etching patterns under particular experimental conditions, it is important to compare the etching patterns resulting from: 1) the dissolution of different polymorphs of the same compound and of a series of related compounds, 2) the dissolution of different faces, 3 ) the action of different specially, selected solvents.

We have undertaken such a systematic study, continuing our previous work. The present contribution reports on the results of a comparative study of the etching patterns on the (010) and the (001)-faces of the crystals of the monoclinic (space group P2 ${ }_{1} / a$ ) and of the orthorhombic (space group Pbca) polymorphs of paracetamol, which differ in the structure of the hydrogenbonded 2D-layers. The etching of faces parallel and normal to the molecular layers was compared. The solutions of acetic anhydride, pyridine, 1,4-dioxane, tetrahydro-1,4-oxazine (morpholine), N-(methyl)-tetrahydro-1,4-oxazine (N-methyl morpholine) (Figure 4) in carbon tetrachloride were used as etching agents. To achieve a selective interaction of a solvent 
molecule with the surface, we decided to take compounds which can bind selectively with the surface atoms. They were chosen among the compounds that are known to form co-crystals with paracetamol, binding selectively the chains and the layers of paracetamol in the structure. ${ }^{16}$ As a reference, thermal etching (sublimation) figures were compared, since these processes depend on the anisotropy of the crystal structure of paracetamol only (no second chemical is involved).<smiles>CC(=O)OC(C)=O</smiles>

$\mathbf{a}$

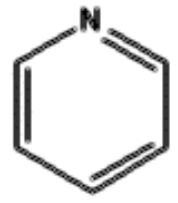

b
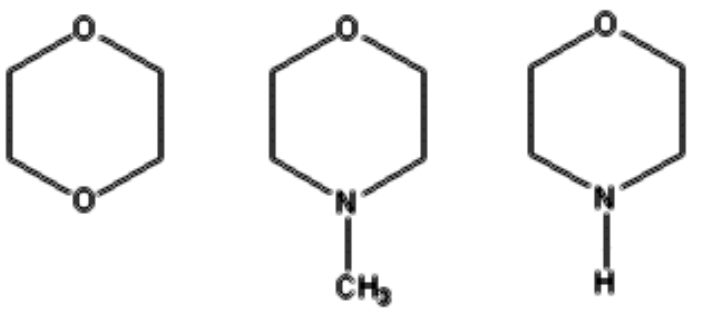

c d e

Figure 4. Molecular structures of the etching agents: a) acetic anhydride, b) pyridine, c) 1,4dioxane, d) tetrahydro-1,4-oxazine (morpholine), e) $N$-(methyl)-tetrahydro-1,4-oxazine ( $N$ methylmorpholine).

\section{Experimental Section}

General Procedures. The crystals of paracetamol I (space group P21/a, $a=12.89(2) \AA, b=$ 9.38(1) $\left.\AA, c=7.11(2) \AA, \mathrm{B}=115.7(2)^{\circ}, \mathrm{Z}=4\right)^{5,7}$ were grown from saturated ethanol solutions by slow cooling from 35 down to $18^{0} \mathrm{C}$ at a cooling rate of $3^{0} \mathrm{C} / 24$ hours. The crystals of paracetamol II (space group Pbca, $a=17.162(3) \AA, b=11.835(8) \AA, c=7.405(1) \AA, Z=8)^{6,14}$ were grown from hot saturated water solutions. ${ }^{21}$ The habit of the crystals is shown in Figure 5. The cell parameters and the face indices were determined using a single-crystal four-circle diffractometer STADI-4 (Stoe, Darmstadt). 


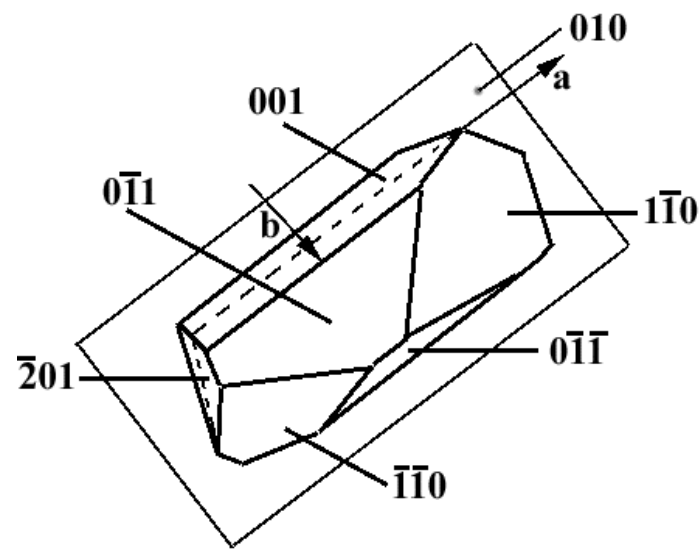

a)

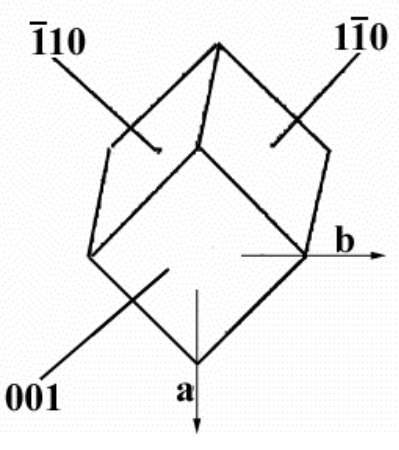

b)

Figure 5. Indices of the faces of the paracetamol polymorphs: a) paracetamol I (P21/a); b) paracetamol II (Pbca).

For paracetamol I, the fresh (010)-faces were obtained by cleaving. The faces (001) could not be obtained by cleaving and were treated as they were grown, being preliminarely washed by carbon tetrachloride. For paracetamol II, the fresh (100)-faces were cleaved. The faces (011) could not be obtained by cleaving and were treated as they were grown, being preliminarely washed by carbon tetrachloride.

Acetic anhydride, pyridine, 1,4-dioxane, tetrahydro-1,4-oxazine, N-(methyl)-tetrahydro-1,4oxazine were obtained from Merck. 10\%-Solutions of acetic anhydride, pyridine, 1,4-dioxane, tetrahydro-1,4-oxazine, $\mathrm{N}$-(methyl)-tetrahydro-1,4-oxazine in carbon tetrachloride were used for etching. The etching time was $30 \mathrm{sec}$. The etching temperature was kept as $20 \pm 2{ }^{\circ} \mathrm{C}$.

Paracetamol was etched thermally at the pressure of $2.34 \mathrm{kPa}$ at the temperature about $20{ }^{\circ} \mathrm{C}$, with am etching time of 40 minutes.

The etching patterns on the faces of the paracetamol crystals were observed using a Neophot2 optical microscope (Karl Zeiss Jena) with a scanning photodevice (Canon G1 2048 x 1536 (3.3 MPix)).

\section{Results}

Etching of the (010) face of paracetamol I and the (001) face of paracetamol II (parallel to molecular layers)

The etching patterns formed on sublimation and at the initial stages of the interaction of the (010) face of paracetamol and (001) face of paracetamol II with various solvents are shown in Figure 6. 


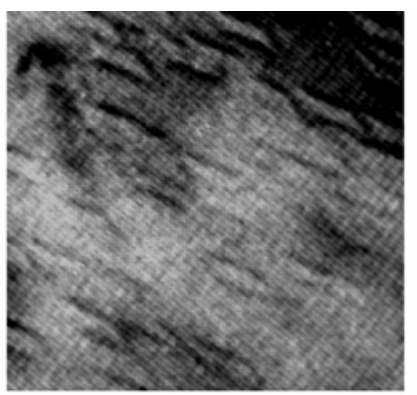

thermal etching
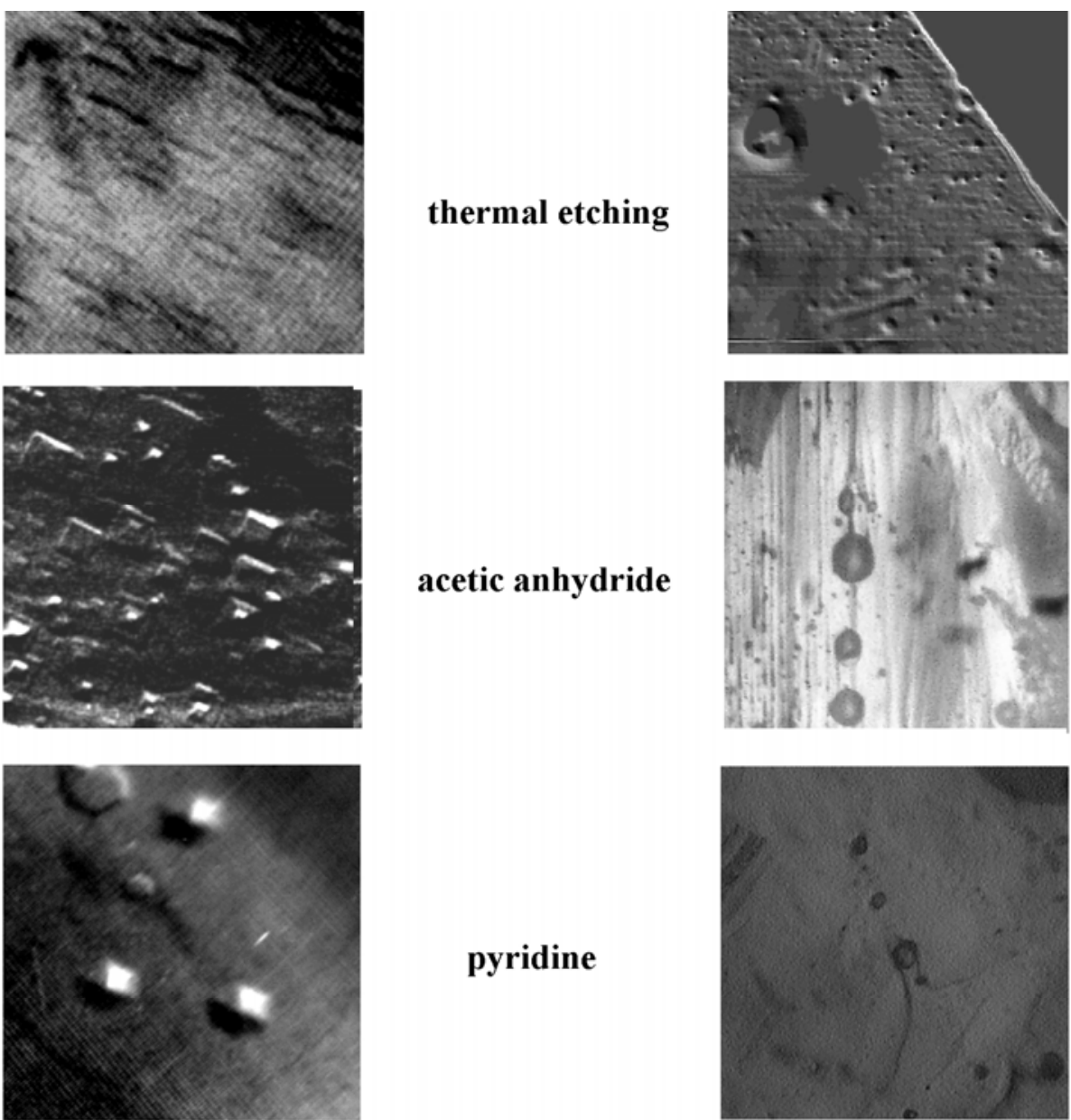

acetic anhydride

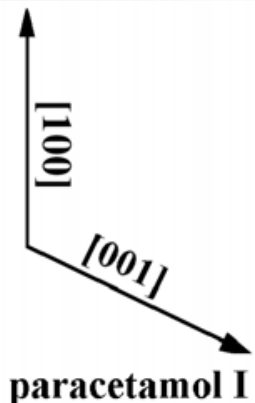

pyridine
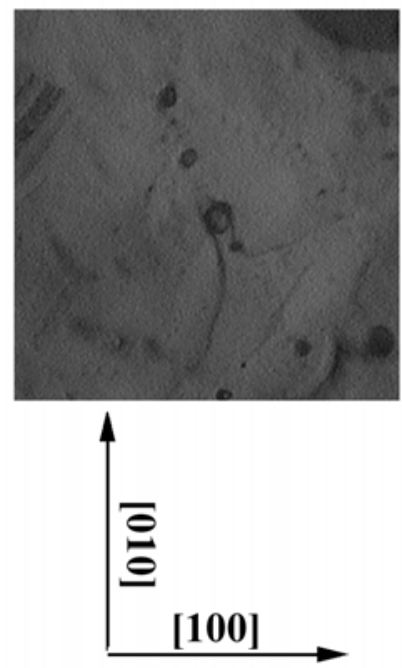

paracetamol II

Figure 6. Optical micrographs of the etching figures at the faces of paracetamol I and paracetamol II parallel to molecular layers (part 1). 


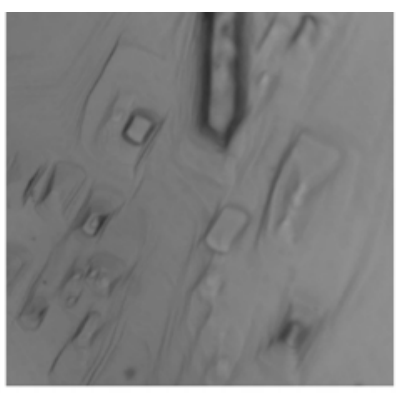

dioxane
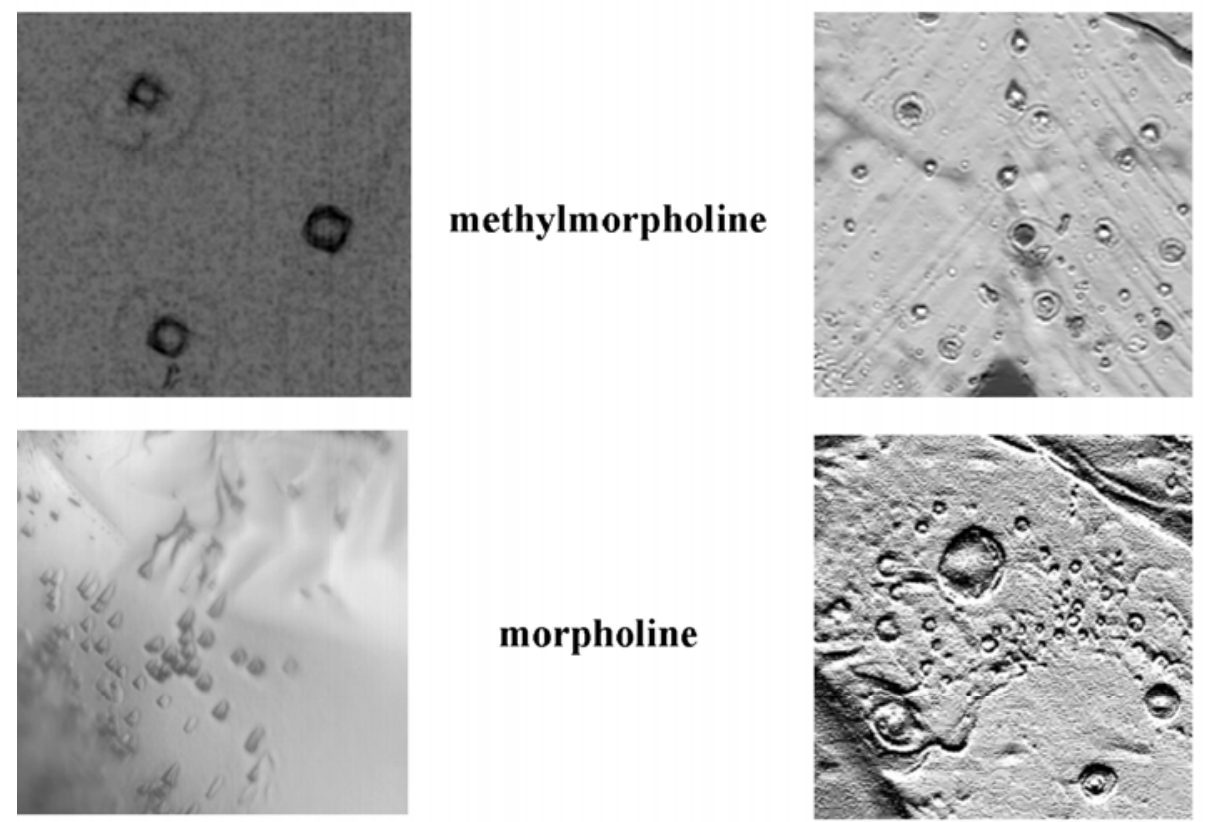

morpholine
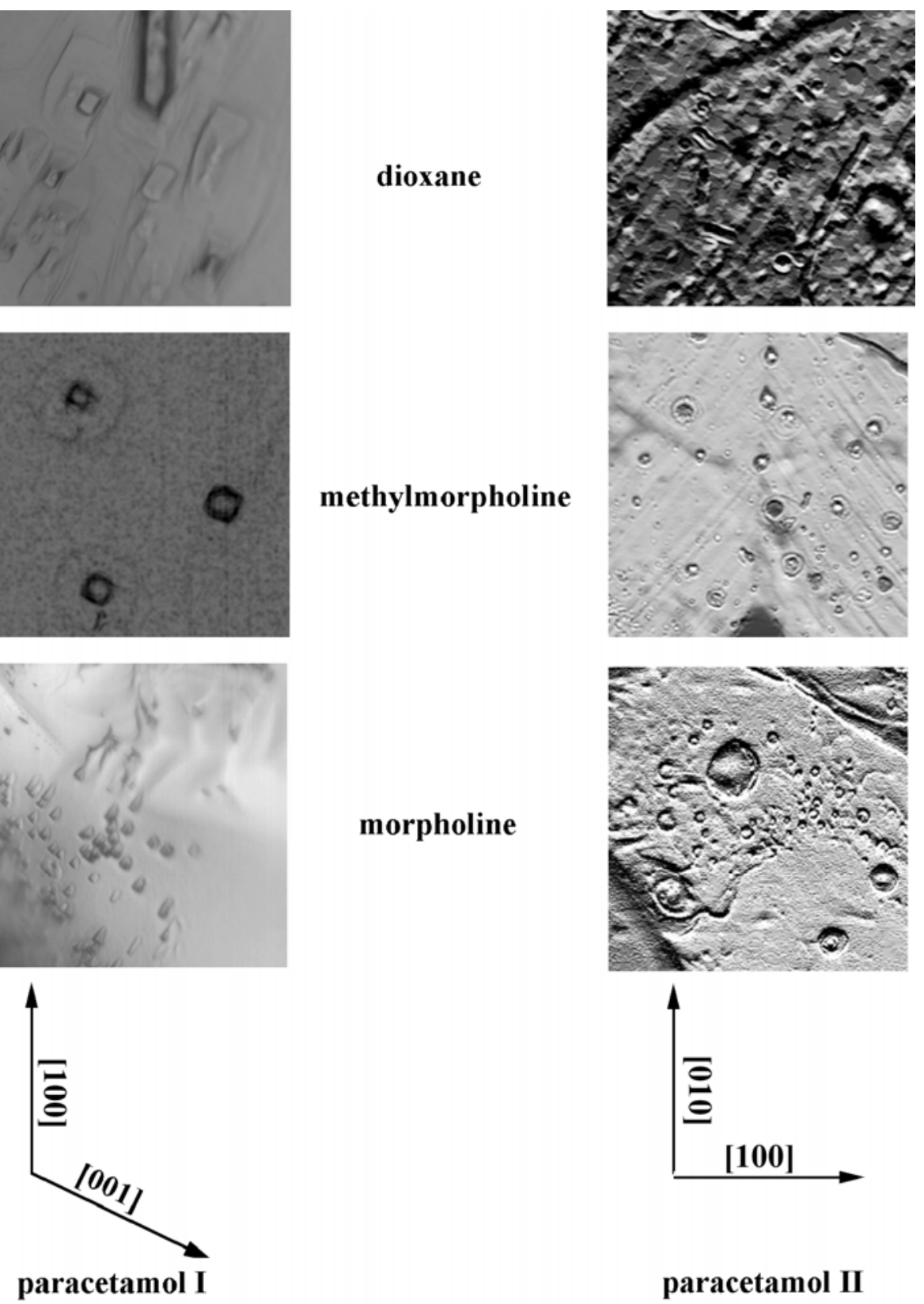

paracetamol II

Figure 6. Optical micrographs of the etching figures at the faces of paracetamol I and paracetamol II parallel to molecular layers (part 2).

During thermal etching, pits with rectangular shapes elongated along [001] were observed for paracetamol I, and it's with rhombic shape with diagonals along [010] and [100] - for paracetamol II. This interaction of paracetamol I with acetic anhydride produced etching pits of a rectangular shape with the diagonal along [100]. Etching by pyridine gave rise to hexagonal etching pits elongated along [100]. Etching the same face by dioxane gave rectangular etching pits diagonal along normally to [001]. After treatment by morpholine and methylmorpholine, the etching pits had the shape diamonds (rhomb) (with diagonal along normally to [001]). 
For paracetamol II, etching by acetic anhydride gave hexagonal pits elongated along [001], etching by dioxane and methylmorpholine gave rhombic etching pits with diagonals along directions [010] and [100], and etching by morpholine and pyridine gave etching pits of spherical shape.

\section{Etching of the (001) face of paracetamol I and (110) face of paracetamol II (normal to molecular layers)}

The shapes of etching pits obtained at the faces of paracetamol I and II normal to molecular layers differed less for thermal treatment and for chemical treatment with various chemicals. The etching pits were elongated along the slip planes. The degree of the anisotropy was different for various reagents. As examples, the etching pits formed after treatment of paracetamol I and paracetamol II with dioxane are shown in Figure 7.
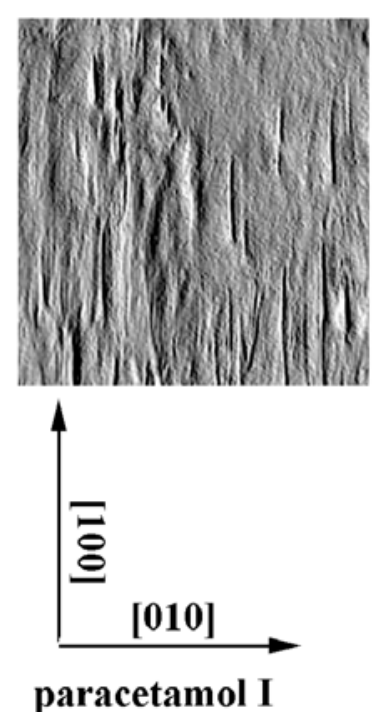
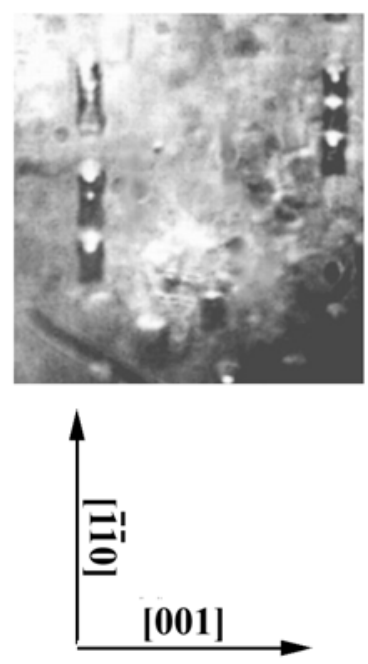

paracetamol II

Figure 7. Optical micrographs of face normal to molecular layers.

\section{Discussion}

To interpret the results of the comparative observations, the following main factors were taken into account:

1. The juxtaposition of the molecules differs from face to face and from one polymorph form to another.

2. The interaction between the molecules of the etching agent and those of paracetamol is selective. The etching agent can bind selectively to a particular functional group, and the juxtaposition of these groups at the surface is pre-determined by the crystal structure.

3. The paracetamol molecules at the surface differ by their activity with respect to the solvent molecules due to their different location. 
These factors manifest themselves differently at different stages of the dissolution, depending on the rate - limiting stage of the process.

In etching, in the simplest approximation, we can distinguish three possible limiting stages: (i) adsorption of the etching agent at the crystal surface, (ii) formation of an intermediate complex between the etching molecule and the molecule of a molecular crystal, and (iii) desorption of this complex from the surface into the solution.

If the etching figures are formed as a result of sublimation, then the two first stages are obviously absent, and the limiting stage is the removal of a paracetamol molecule from a site at the surface. For the thermal etching of faces parallel to the molecular layers, the anisotropy of the process can be expected to depend solely on the anisotropy of the crystal structure. The layers in the paracetamol II are flat, and the etching pits formed on sublimation have a nearly square shape, thus reflecting the symmetry of the molecular disposition in the structure, as it is usually observed when etching crystals of ionic salts. ${ }^{1}$ The layers in the structure of paracetamol I, however, are not flat, but corrugated, and have "hidges" and "valleys" (Figure 8). The molecules at the "hills" can be expected to be attacked more easily by a solvent molecule than those in the "valleys". As a result, one can expect the formation of etching pits elongated along the "hidge", and this is what was really observed in the experiments on thermal etching of paracetamol I. Thermal etching of faces normal to molecular layers is determined by interlayer interactions. In the two polymorphs the interactions between the layers are van der Waals, no hydrogen bonds are formed. Therefore, the differences in the anisotropy of etching pits formed on thermal and on chemical etching, and between the two polymorphs are less pronounced than when etching faces parallel to the layers.

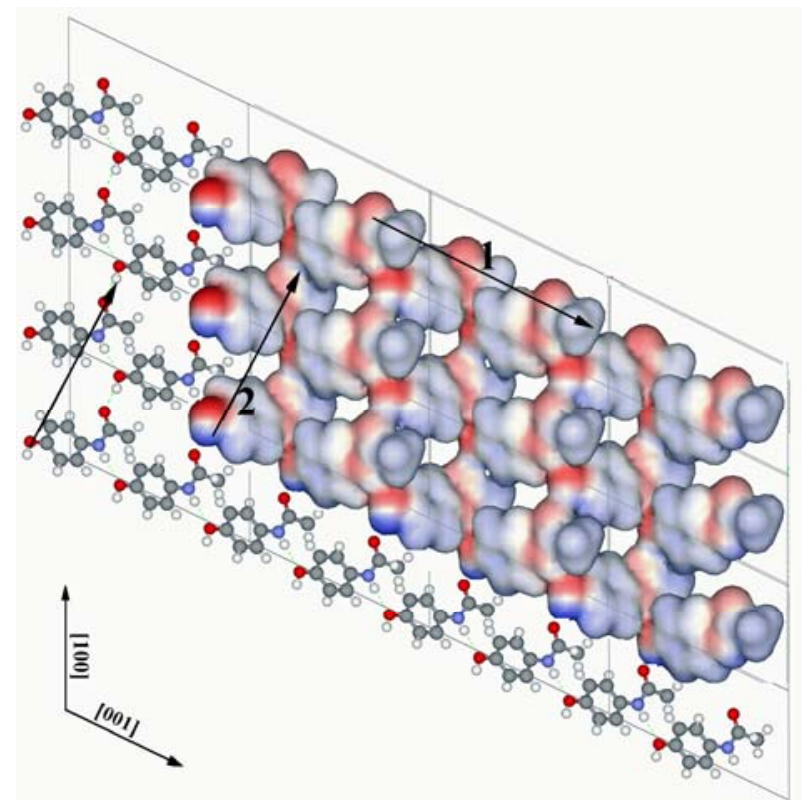

Figure 8. Idealized structures of (010)-faces of paracetamol I parallel to the molecular layers (1 - direction of the development of reaction front due to sublimation, 2 - direction of the development of the reaction front due to the sorption of a bis-donor solvent molecule). 
The differences in the anisotropy of chemical etching are determined not only by the anisotropy of crystal structures, but also by the different selectivity of the interaction of solvent molecules (various reagents) with different molecules at the paracetamol surface. This selectivity can be correlated simply with the donor capacity of the etchant molecules (characterized, e.g., by Marcus coefficient ${ }^{22}$ ), and this is what was done in our previous work correlating donor numbers of the etching solvents with the anisotropy of the etching pits formed. ${ }^{2,3,11,12}$ Alternatively, the interpretation can be based on a more detailed consideration of the possibility of the formation of an intermediate complex between a surface molecule and an etchant molecule ("molecular recognition concept", "key-and-lock" principle). ${ }^{23}$ In this case, not only the donor properties, but also the disposition of the neighbouring molecules at the paracetamol surface (Table 1) and the geometry of the etchant molecules, in particular the number of the potentially active centers and their location (Table 2) must be important.

Table 1. Distances between the centers of the molecules (1) and the N...O (H...O) distances in the $\mathrm{N}(\mathrm{O}) \mathrm{H} . . . \mathrm{O}$ and $\mathrm{OH} . . \mathrm{O}$ hydrogen bonds (2) in the structure of paracetamol $\mathrm{I}^{7}$ (numeration of molecules is explained in Figure 2)

\begin{tabular}{cccc}
\hline Molecule number & Distances 1, $\AA$ & Distances 2, $\AA$ & Type of hydrogen bond \\
\hline $1-2(5)$ & 5.92 & $2.93(2.05)$ & $\mathrm{N}(2)-\mathrm{H}--\mathrm{O}(1)$ \\
$1-4(7)$ & 6.17 & $2.66(1.80)$ & $\mathrm{O}(1)-\mathrm{H}-\mathrm{O}(2)$ \\
$1-3(6)$ & 6.17 & - & -
\end{tabular}

One can expect the etchant molecules to attack first the most weakly bound molecules at the surface of paracetamol I, namely the molecules at the peaks of the corrugated "ridges". One can also expect that during chemical etching the neighboring molecules will be also activated as a result of the interaction of the etching molecule with a paracetamol molecule at the surface, so that several neighbors can be involved in the dissolution at a reaction site. The interaction between a solvent molecule and a surface paracetamol molecule involves the formation of hydrogen bonds with the oxygen and nitrogen centers of the paracetamol molecules. Therefore, the interaction should be stronger the higher is the Marcus coefficient (M) of the etching agent molecule. Actually, in agreement with our previous observations, ${ }^{3}$ with the increase of the donor number of a reagent, we have observed a decrease in the selectivity of the process, resulting in a change in the shape of the etching pits: from the lines elongated along [001] towards square pits. There was a tendency for the propagation of the etching front along [100] in case of acetic anhydride $(M=0.27)$. Hexagonal pits were observed in case of etching by pyridine $(M=0.85)$, when the interaction between the pyridine and paracetamol molecules could be supposed to cause the activation of six molecules surrounding the attacked molecule.

If the etchant molecules have two active centers, the mutual orientation of the layers of the paracetamol molecule and the solvent molecule can be important. Thus, the molecules of 
morpholine and methylmorpholine can be expected to bind simultaneously to two paracetamol molecules in layers, if we consider the location of molecules in their co-crystals with paracetamol. $^{20}$ This can explain why during etching of paracetamol by morpholine and methylmorpholine the reaction front is directed normally to [001].

During etching of paracetamol II, the differences in the shapes of the etching pits formed at the faces parallel to the molecular layers, and the sharpness of their angles, are less pronounced, due to weaker hydrogen bonds ${ }^{18}$ and, hence, a lower selectivity of dissolution and a less pronounced anisotropy of etching. The almost square shape of the etching pits formed at the face normal to the molecular layers, in case of etching paracetamol II with dioxane, morpholine, and methylmorpholine (molecules with the two donor centers) reflects the location of the neighbouring paracetamol molecules at the face (110) of paracetamol II activated during dissolution.

\section{Conclusions}

We have studied the topochemistry of the initial dissolution stages of paracetamol polymorphic modifications. The shapes of the etching pits were shown to depend on the paracetamol crystal structure and on the structure and donor strength of etching reagents.

\section{Acknowledgements}

The research described in this publication was supported in part by Award No. NO-008-X1 of the U.S. Civilian Research \& Development Foundation for the Independent States of the Former Soviet Union (CRDF), by the grant Y-0069 (Integration) of the Russian Ministry of Education, by the grant 03-03-06092 of the Russian Foundation of Basic Research, and by the Program of the RAS "Fundamental studies in medicine", project 11.2. The authors are grateful to Prof. E.V. Boldyreva for valuable discussions.

\section{References}

1. Sangwal, K. In Etching of Crystals; North-Holland: Amsterdam, 1987.

2. Vasilchenko, M. A.; Shakhtshneider, T.P.; Naumov, D. Yu.; Boldyrev, V.V. J. Therm. Analys. Calorim. 1999, 57, 157.

3. Boldyrev, V.V.; Vasilchenko, M. A.; Shakhtshneider, T. P.; Naumov, D.Yu.; Boldyreva, E.V. Solid State Ionics 1997, 101-103 (Pt. 2), 869-864.

4. Shekunov, B.Yu.; Grant, D.J.W. J. Phys. Chem. B 1997, 101, 3973.

5. Nichols, G.; Frampton, C.S. J. Pharm. Sci. 1998, 87, 684. 
6. Haisa, M.; Kashino, S.; Maeda, H. Acta Crystallogr. 1974, B 30, 2510.

7. Vasilchenko, M.A.; Shakhtshneider, T.P.; Naumov, D.Yu.; Boldyrev, V.V. J. Pharm. Sci. 1996, 85, 929.

8. Boldyreva, E.V.; Shakhtshneider, T.P.; Ahsbahs, H.; Sowa, H.; Uchtmann, H. J. Therm. Anal. 2002, 66, 437.

9. Williams, F.R; Heng, J.Y.Y. Book of Abstracts, PhandTA 8, Ascona, Monte Verita, 2004, SC 15.

10. Vasilchenko, M.A.; Shakhtshneider, T.P.; Naumov, D.Yu.; Boldyreva, E.V; Boldyrev, V.V. Doklady Akad. Nauk (Proc. Russian Acad. Sci.) 1996, 350, 777.

11. Vasilchenko, M.A.; Shakhtshneider, T.P.; Naumov, D.Yu.; Boldyrev, V.V. Doklady Akad. Nauk (Proc. Russian Acad. Sci.) 1999, 366, 210.

12. Ivashchenko, V.E.; Boldyrev, V.V.; Zakharov, Yu.A.; Shakhtshneider, T.P Ermakov, A.E.; Krasheninin, V.I. Mat. Res. Innovat. 2002, 5, 214.

13. Shakhtshneider, T.P.; Boldyreva, E.V.; Vasilchenko, M.A.; Ahsbahs, H.; Uchtmann, H. Russ. J. Struct. Chem. 1999, 40, 1101.

14. Shakhtshneider, T.P.; Boldyreva, E.V.;Vasilchenko, M.A.; Ahsbahs, H.; Uchtmann, H. Doklady Akad. Nauk (Proc. Russian Acad. Sci.) 1998, 363, 783.

15. Boldyreva, E.V.; Shakhtshneider, T.P.; Vasilchenko, M.A.; Ahsbahs, H.; Uchtmann, H. Acta Crystallogr. 2000, B 56, 299.

16. Boldyreva, E.V.; Shakhtshneider, T.P.; Ahsbahs, H.; Uchtmann, H.; Burgina, E.B.; Baltakhinov, V.P. Polish J. Chem. 2002, 76, 1333.

17. Li, T.; Morris, K.R.; Park, K. J. Phys. Chem. 2000, B 104, 2019.

18. Oswald, J.D.H.; Allan, D. R.; McGregor, P. A.; Motherwell, W.D.S.; Parsons, S.; Pulham, C. R. Acta Crystallogr. 2002, B 58, 1057.

19. Mikhailenko, M.A. J. Crystal Growth. 2004, 265, 616.

20. Drebushchak, T.N.; Boldyreva, E.V. Z. Kristallogr. 2004, 219, 506

21. Markus, Y. J. Sol. Chem. 1984, 13599.

22. Weissbuch, I.; Popovitz-Biro, R.; Lahav, M.; Leiserowitz, L. Acta Crystallogr. 1995, B 51, 115 . 